UNIVERSIDADE ESTADUAL DE FEIRA DE SANTANA

Autorizada pelo Decreto Federal $n^{\circ} 77.496$ de 27/04/76

Recredenciamento pelo Decreto $n^{\circ} 17.228$ de $25 / 11 / 2016$

PPPG

PRÓ-REITORIA DE PESQUISA E PÓS-GRADUAÇÃO

COORDENAÇÃO DE INICIAÇÃO CIENTÍFICA

XXIII SEMINÁRIO DE INICIACCÃO CIENTÍFICA DA UEFS

SEMANA NACIONAL DE CIENTÍFICA E TECNOLÓGICA - 2019

\title{
MAMÍFEROS TERRESTRES DA SERRA DA JIBOIA, RECÔNCAVO BAIANO
}

\author{
Bruna da Silva Sampaio ${ }^{1}$; Téo Veiga de Oliveira ${ }^{2}$ \\ 1. Bolsista PIBIC/FAPESB, Graduanda em Bacharelado em Ciências Biológicas, Universidade Estadual de Feira de \\ Santana, e-mail: sampaiobruna55@yahoo.com \\ 2. Orientador, DCBio, Universidade Estadual de Feira de Santana, e-mail: teovoli@yahoo.com.br
}

PALAVRAS-CHAVE: pequenos mamíferos; mamíferos de maior porte; armadilhas fotográficas.

\section{INTRODUÇÃO}

A Serra da Jiboia compreende uma porção de Mata Atlântica, localizada no Recôncavo Sul da Bahia (Blengini et al., 2015). Dentre os animais que habitam esta região, um dos grupos que tem o maior apelo conservacionista são os mamíferos. Alguns poucos trabalhos sobre este tema já foram feitos na região (ver um sumário em Oliveira et al., 2018) e, neles, cerca de 80 mamíferos de ordens diversas já tiveram sua ocorrência atribuída para a área. Dentre eles, encontram-se formas tradicionalmente ditas de pequeno porte, particularmente didelfimorfios (marsupiais) e roedores.

Estes pequenos mamíferos estão entre as formas mais diversas no Brasil (Paglia et al., 2012) e a eles pertencem algumas das espécies que prestam o maior número de serviços ecossistêmicos (são dispersores sementes, polinizadores, consumidores de invertebrados e plantas e presas de vertebrados maiores (Emmons \& Feer, 1997; Reis et al., 2011). Também as formas de mamíferos terrestres de maior porte desempenham um importante papel nos vários níveis da organização dos ecossistemas, principalmente no controle do tamanho das populações de suas presas, no caso de mamíferos carnívoros, e na regeneração das florestas, quando levados em consideração as espécies herbívoras (Emmons \& Feer, 1997; Abreu Jr. \& Kohler, 2009).

O objetivo do presente trabalho foi dar continuidade ao inventário de mamíferos terrestres que vem sendo desenvolvido pela equipe da Divisão de Mamíferos do Museu de Zoologia da UEFS na Serra da Jiboia, Recôncavo da Bahia.

\section{MATERIAL E MÉTODOS}

A Serra da Jiboia estende-se por cinco municípios: Elísio Medrado, Santa Teresinha, Castro Alves, Varzedo e São Miguel das Matas. Com aproximadamente 9.000ha, inclui o mais significativo remanescente de Mata Atlântica da região, com aproximadamente 5.600ha (Blengini et al., 2015). As expedições de campo envolveram a instalação de três armadilhas fotográficas, em pontos da mata que sugeriam o trânsito de mamíferos (presença de cursos d'água e de trilhas produzidas por animais, por exemplo). Próximo a cada armadilha foram amarradas, em árvores ou colocadas no 
chão, iscas odoríferas (pedaços de frutas, sardinha, bacon, paçoca etc.) visando atrair animais para a área de ação das câmeras (O’Connel et al., 2011).

Duas expedições de campo foram realizadas, ambas na Reserva Jequitibá, localizada no município de Elísio Medrado, a primeira compreendendo o período entre o fim de agosto e meio de dezembro de 2018, onde as armadilhas foram revisadas e retiradas dos pontos de amostragem e, no mesmo dia, realocadas para novos pontos, compreendendo o segundo período de amostragem entre o meio de dezembro de 2018 e início de junho de 2019, totalizando 272 dias de amostragem efetiva, além dos dias de instalação das armadilhas onde as mesmas eram testadas e revisadas, também foram efetuadas expedições de campo durante os períodos de amostragem para revisar e verificar seu funcionamento.

A Coleção de Mamíferos do Museu de Zoologia conta com vários indivíduos oriundos da Serra da Jiboia. Estes espécimes foram revisados e foram adicionadas as informações obtidas na ocasião das coletas, como os dados biométricos, caso ainda fosse necessário, os quais auxiliam na identificação taxonômica, juntamente com literatura específica sobre os táxons (e.g. Emmons \& Feer, 1997; Bonvicino et al., 2008; Reis et al., 2011).

\section{RESULTADOS E DISCUSSÃO}

Ao fim do período amostral 10 espécies foram registradas (além de cães domésticos) (Tabela 1; Figura 1). A lista mais completa da mastofauna presente na Serra da Jiboia (Oliveira et al., 2018), possui 84 espécies e os dados aqui apresentados representam espécies já registradas, mesmo assim, Leopardus emiliae e Eira barbara foram registradas pela primeira vez na Reserva Jequitibá, aumentando o número de registros dos mesmos para a área em um novo ponto amostral. O número de espécies registradas no presente estudo representa $18,2 \%$ das espécies de mamíferos terrestres presentes na Serra da Jiboia, e é bastante significativo quando comparamos estudos com maior período de amostragem de armadilhas fotográficas em outras áreas (e.g. Aximoff et al., 2017), ressaltando que a riqueza de espécies presente na Serra da Jiboia é muito significante e precisa ser conservada.

Tabela 1. Lista de espécies registradas.

\begin{tabular}{ll}
\hline \hline DIDELPHIMORPHIA & Felidae \\
Didelphidae & Leopardus pardalis (Linnaeus, 1758) \\
Metachirus nudicaudatus (Desmarest, 1817) & Leopardus emiliae (Thomas, 1914) \\
RODENTIA & Procyonidae \\
Echimyidae & Nasua nasua (Linnaeus, 1766) \\
Trinomys sp. & Procyon cancrivorus (G. Cuvier, 1798) \\
Cuniculidae & Mustelidae \\
Cuniculus paca (Linnaeus, 1766) & Eira barbara (Linnaeus, 1758) \\
PILOSA & CETARTIODACTYLA \\
Myrmecophagidae & Tayassuidae \\
Tamandua tetradactyla (Linnaeus, 1758) & Pecari tajacu (Linnaeus, 1758) \\
\hline \hline
\end{tabular}




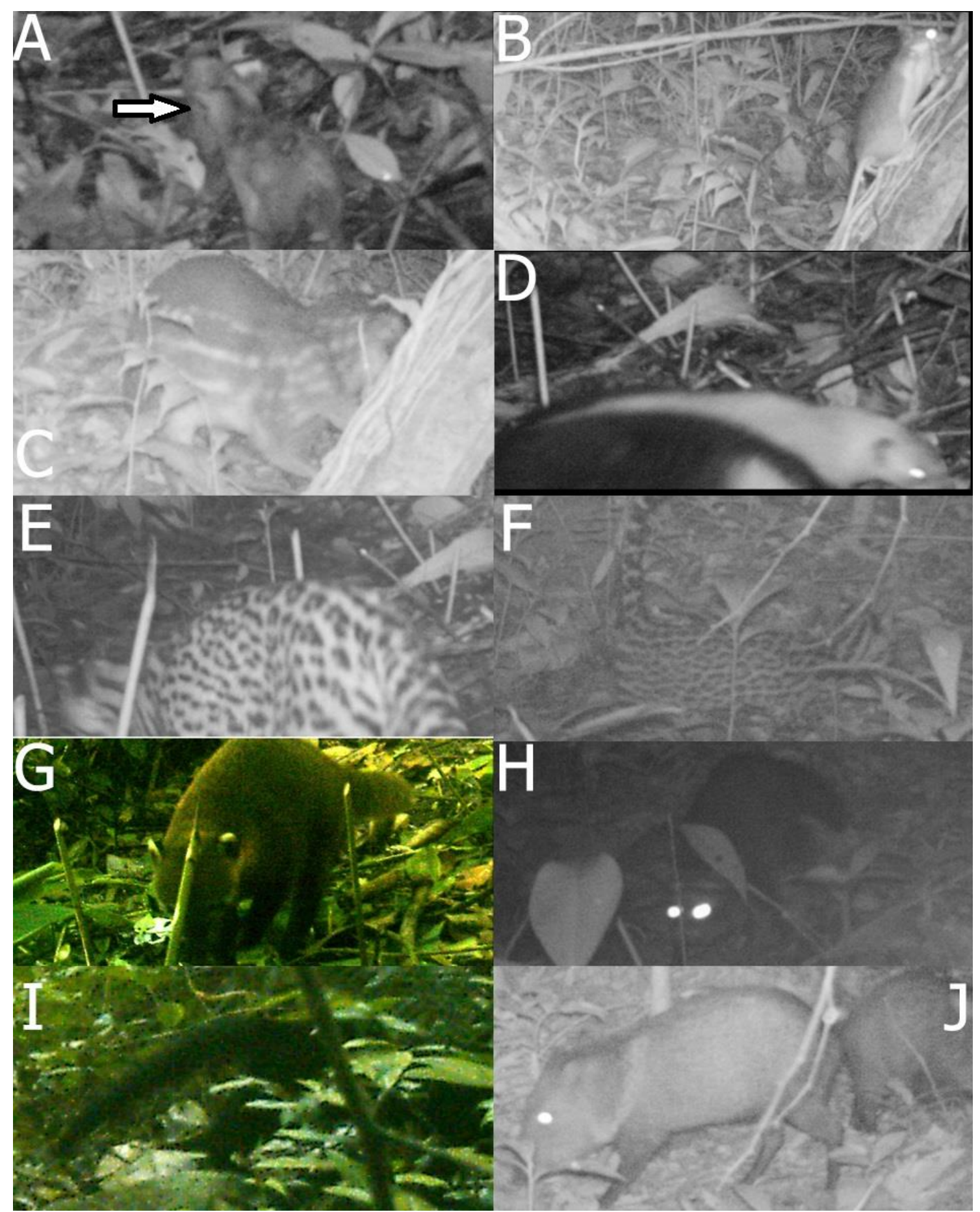

Figura 1. Imagens das espécies registradas. A, Metachirus nudicaudatus; B, Trinomys sp.; C, Cuniculus paca; D, Tamandua tetradactyla; E, Leopardus pardalis; F, Leopardus emiliae; G, Nasua nasua; H, Procyon cancrivous; I, Eira barbara; J, Pecari tajacu.

\section{CONSIDERAÇÕES FINAIS}

O uso de armadilhas fotográficas é uma ótima ferramenta para registros de mamíferos, especialmente os de maior porte, os quais são dificilmente capturados, permitindo assim inventários mais completos da mastofauna. O registro de Leopardus emiliae, espécie endêmica e recentemente revalidada, e a significativa quantidade de espécies de mamíferos registradas na Serra da Jiboia nos últimos anos reforça o quão 
importante e urgente é a aprovação de um plano de conservação para a região, de modo a preservar este importante refúgio para muitas espécies.

\section{REFERÊNCIAS}

ABREU JR., E.F. \& KÖHLER, A. 2009. Mammalian fauna of medium and large sized in the RPPN of UNISC, RS, Brazil. Biota Neotropica, 9(4):169-174.

AXIMOFF, I., CRONEMBERGER, C., \& PEREIRA, F. 2017. Amostragem de longa duração por armadilhas fotográficas dos mamíferos terrestres em dois parques nacionais no estado do Rio de Janeiro. Oecologia Australis, 19(1).

BLENGINI, I.A.D.; CINTRA, M.A.M.U.; CUNHA, R.P.P. \& CAIAFA, A.N. 2015. Proposta de Unidade de Conservação da Serra da Jiboia. Salvador, Grupo Ambientalista da Bahia.

EMMONS, L. \& FEER, F. 1997. Neotropical rainforest mammals. A field guide. Second edition. Chicago: The University of Chicago Press.

O'CONNELL, A.F.; NICHOLS, J.D. \& KARANTH, K.U. 2011. Camera traps in animal ecology: methods and analyses. New York: Springer, 271p.

OLIVEIRA, T.V.; SAMPAIO, B.S.; SILVA, W.H.; CALIXTO, B.M.; CARVALHO, M.S.; BORGES, L.S. \& SCHERER, C.S. 2018. A fauna de mamíferos voadores e não-voadores da Serra da Jiboia, Bahia, Brasil. Boletim da Sociedade Brasileira de Mastozoologia, 82: 68-88.

PAGLIA, A.P., FONSECA, G.A.B. DA, RYLANDS, A. B., HERRMANN, G., AGUiAR, L. M. S., CHIAREllo, A. G., LEITE, Y. L. R., COSTA, L. P., SICILIANO, S., KIERULFF, M. C.M., MENDES, S. L., TAVARES, V. DA C., MITTERMEIER, R. A. \& PATTON J. L. 2012. Lista Anotada dos Mamíferos do Brasil. 2a Edição. Occasional Papers in Conservation Biology, 6:176.

REIS, N.R.; PERACHI, A.L.; PEDRO, W.A. \& LIMA, I.P. 2011. Mamíferos do Brasil. 2ed. Londrina, Os autores. 